\title{
Coupled Flow Field Simulations of Charring Ablators with Nonequilibrium Surface Chemistry
}

\author{
Hicham Alkandry* and Iain D. Boyd ${ }^{\dagger}$ \\ Department of Aerospace Engineering, University of Michigan, Ann Arbor, MI \\ Alexandre Martin ${ }^{\ddagger}$ \\ Department of Mechanical Engineering, University of Kentucky, Lexington, KY
}

\begin{abstract}
This paper describes the coupling of a Navier-Stokes solver to a material response code to simulate nonequilibrium gas-surface interactions. The Navier-Stokes solver used in this study is LeMANS, which is a three-dimensional computational fluid dynamics code that can simulate hypersonic reacting flows including thermo-chemical nonequilibrium effects. The material response code employed in this study is MOPAR, which uses the one-dimensional control volume finite-element method to model heat conduction and pyrolysis gas behavior. This coupling is demonstrated using a test case based on the Stardust sample return capsule. Coupled simulations are performed at three different trajectory conditions. The effects of the pyrolysis gas chemistry are evaluated by assuming that the gas is either in chemical equilibrium or composed entirely of non-reacting phenol. The results show that the non-reacting pyrolysis gas assumption produces higher convective heat fluxes, surface temperatures, and mass blowing rates. These effects are mainly due to the composition of the pyrolysis gas. The additional species produced by the pyrolysis gas in the chemical equilibrium case react with oxygen and nitrogen atoms in the gas-phase. This results in fewer atoms participating in the exothermic surface reactions, which reduces the heat transfer to the vehicle.
\end{abstract}

\section{Nomenclature}

$\begin{array}{ll}D_{k} & \text { Mass diffusion coefficient of species } k, \mathrm{~m}^{2} / \mathrm{s} \\ h & \text { Enthalpy, J/kg } \\ \dot{m} & \text { Mass blowing rate, } \mathrm{kg} / \mathrm{m}^{2} / \mathrm{s} \\ M_{k} & \text { Molar weight of species } k, \mathrm{~kg} / \mathrm{mol} \\ N B & \text { Number of bulk-phase species } \\ p & \text { Pressure, } \mathrm{Pa} \\ \dot{s} & \text { Recession rate, } \mathrm{m} / \mathrm{s} \\ T_{t r} & \text { Translational/rotational temperature, } \mathrm{K} \\ T_{v e} & \text { Vibrational/electronic/electron temperature, } \mathrm{K} \\ v & \text { Velocity, } \mathrm{m} / \mathrm{s} \\ \dot{w}_{k} & \text { Molar production rate of species } k, \mathrm{~mol} / \mathrm{m}^{2} / \mathrm{s} \\ Y_{k} & \text { Mass fraction of species } k \\ \rho & \text { Density, kg/m }{ }^{3} \\ s u b s c r i p t s & \\ c & \text { Char } \\ g & \text { Pyrolysis gas } \\ n & \text { Conditions at the first cell away from the wall } \\ w & \text { Conditions at the wall }\end{array}$

* Research Fellow, Member AIAA.

$\dagger$ James E. Knott Professor of Engineering, Fellow AIAA.

${ }_{\ddagger}^{\ddagger}$ Assistant Professor, Associate Faculty at the Center for Computational Science, Senior Member AIAA. 


\section{Introduction}

P Lanetary entry vehicles are required to sustain very high heat loads when entering an atmosphere. 1 For this reason, many vehicles employ charring thermal protection system (TPS) materials. Accurate simulations of the aerothermal environment expected during re-entry for these vehicles may need to account for complicated physical processes, such as nonequilibrium surface chemistry and the behavior of pyrolysis gases emitted from the ablative TPS. One way to account for these complex processes is by coupling a flow field solver that can simulate the flow around the re-entry vehicle and a material response code that can model the pyrolysis process and heat conduction within the TPS.

The goal of this study is to couple a viscous Navier-Stokes solver to an existing material response code. The flow field solver employed in this study is the LeMANS computational fluid dynamics (CFD) code. ${ }^{1,2}$ LeMANS is developed at the University of Michigan and has been used to simulate hypersonic reacting flows. Recently, a finite-rate surface chemistry module has been implemented in LeMANS in order to model nonequilibrium chemical processes that may occur at the surface of a re-entry vehicle. ${ }^{3}$ The material response code used in this study is called MOPAR. ${ }^{4-6}$ This code has been developed at the University of Michigan and the University of Kentucky and can model heat conduction and pyrolysis gas behavior with inner decomposition. This new coupled capability is demonstrated using a test case based on the re-entry conditions of the Stardust sample return capsule.

The paper is presented as follows. The first section describes the coupling approach used in this study. Details regarding the Navier-Stokes solver, the material response code, and the coupling aspects are provided. In the second section of this paper, the capabilities of this coupled method are demonstrated using a test case based on the Stardust sample return capsule. The effects of the pyrolysis gas mixture composition are evaluated by comparing the numerical results obtained using two different assumptions. The first approach assumes that the pyrolysis gas is in chemical equilibrium, while the second approach assumes that the pyrolysis gas is chemically "frozen" and does not react with any gas-phase species. The last section of the paper presents conclusions drawn from this study and possible future research directions.

\section{Coupling Approach}

\section{A. Navier-Stokes Solver}

LeMANS is a three-dimensional, parallel code that solves the laminar Navier-Stokes equations on unstructured computational grids including thermo-chemical nonequilibrium effects. In LeMANS, the flow is modeled assuming that the continuum approximation is valid. For this study, it is also assumed that the translational and rotational energy modes can be described by a single temperature, $T_{t r}$, and that the vibrational, electronic, and electron translational energy modes are described by a different temperature $T_{v e}$. The mixture transport properties are calculated using Wilke's semi-empirical mixing rule ${ }^{7}$ with species viscosities computed using Blottner's curve fits ${ }^{8}$ and species thermal conductivities determined using Eucken's relation. ${ }^{9}$ In this study, the species mass diffusion coefficients are calculated assuming a constant Lewis number of 1.4. The mass diffusion fluxes of heavy particles are modeled using a modified version of Fick's law ${ }^{10}$ which enforces the requirement that the fluxes sum to zero, and the diffusion flux of electrons is calculated assuming ambipolar diffusion.

In LeMANS, the set of governing equations are solved using the finite-volume method applied to unstructured grids with second-order spatial accuracy. LeMANS can simulate two-dimensional and axisymmetric flows using any mixture of quadrilateral and triangular mesh cells, and three-dimensional flows using any mixture of hexahedra, tetrahedra, prisms, and pyramids. A modified Steger-Warming Flux Vector Splitting scheme $^{11}$ is used to discretize the inviscid fluxes across cell faces, which is less dissipative and produces better results in boundary layers compared to the original scheme. The viscous terms are computed using cell-centered and nodal values. The viscous stresses are modeled assuming a Newtonian fluid and Stokes' hypothesis, and the heat fluxes are modeled according to Fourier's law for the two temperatures. Mesh movement capabilities have been implemented in LeMANS to model surface recession for ablative heat-shields. ${ }^{6,12}$ The method used in LeMANS solves the discrete form of the geometric conservation laws, which state the balance between the relevant geometric parameters. ${ }^{13}$ This method is valid for both explicit and implicit schemes and retains the order of accuracy of the CFD model. LeMANS is parallelized using METIS ${ }^{14}$ to partition the computational mesh, and the Message Passing Interface (MPI) to communicate the necessary information between processors. 
Nonequilibrium chemical processes at the surface of a re-entry vehicle are modeled in LeMANS using the Finite-Rate Surface Chemistry (FRSC) Module, originally developed by Marschall and MacLean. ${ }^{15,16}$ The FRSC Module allows for the definition of three distinct environments: gas, surface, and bulk. The surface and bulk environments can have any number of phases, which represent physically and chemically distinct regions. The gas environment can only have a single phase. Surface reactions may only occur at active sites that can either be empty or filled with an adsorbed species on a specific surface phase. The total number of reaction sites is conserved and sites may neither be created nor destroyed. The number of reaction sites (i.e. site density) is a property of the surface and is defined as a user input. The FRSC Module allows the specification of several different reaction types. These include,

$\begin{array}{llrl}\text { Adsorption/Desorption: } & \mathrm{A}+(\mathrm{s}) \rightleftharpoons \mathrm{A}(\mathrm{s}) \\ \text { Eley-Rideal: } & \mathrm{A}+\mathrm{B}(\mathrm{s}) \rightleftharpoons \mathrm{AB}+(\mathrm{s}) \\ \text { Langmuir-Hinshelwood: } & \mathrm{A}(\mathrm{s})+\mathrm{B}(\mathrm{s}) \rightleftharpoons \mathrm{AB}+(\mathrm{s}) \\ \text { Sublimation/Condensation: } & (\mathrm{s})+\mathrm{A}(\mathrm{b}) \rightleftharpoons \mathrm{A}+(\mathrm{s})\end{array}$

In the previous examples, (s) represents an empty active site, $\mathrm{A}(\mathrm{s})$ is an adsorbed particle, and $\mathrm{A}(\mathrm{b})$ is a bulk species. The FRSC Module also allows for the specification of several other reaction processes that do not strictly fit into the categories given above (e.g. oxidation and nitridation). Additional details regarding the FRSC Module are provided in Refs. 15 and 16.

\section{B. Material Response Code}

The material response code used in this study is MOPAR and has been developed at the University of Michigan and the University of Kentucky. ${ }^{4-6}$ MOPAR is based on a code developed at the Sandia National Laboratories. ${ }^{17,18}$ It employs the one-dimensional control volume finite-element method (CVFEM) to model heat conduction and pyrolysis gas behavior with inner decomposition. MOPAR computes the surface temperature and pyrolysis gas mass blowing rate by solving the following governing equations:

Mixture Energy Equation:

$$
\frac{d}{d t} \int_{c v} \rho E d V-\int_{c s} \rho h v_{c s} d A+\int_{c s} \phi \rho_{g} h_{g} v_{g} d A+\int_{c s} \dot{q}^{\prime \prime} d A=0
$$

Solid-Phase Continuity Equation:

$$
\frac{d}{d t} \int_{c v} \rho_{s} d V-\int_{c s} \rho_{s} v_{c s} d A-\int_{c v} \dot{m}_{s}^{\prime \prime \prime} d V=0
$$

Gas-Phase Continuity Equation:

$$
\frac{d}{d t} \int_{c v} \phi \rho_{g} d V-\int_{c s} \phi \rho_{g} v_{c s} d A+\int_{c s} \phi \rho_{g} v_{g} d A-\int_{c v} \dot{m}_{g}^{\prime \prime \prime} d V=0
$$

Momentum Equation:

$$
\frac{\partial p}{\partial x}=-\frac{\mu}{K} \phi v_{g}
$$

where $V$ is the volume, $A$ is the area, $E$ is the total energy, $\dot{q}^{\prime \prime}$ is the internal heat flux, $\dot{m}^{\prime \prime \prime}$ is the volumetric mass source term, $\phi$ is the porosity, and $p$ is pressure. The subscript $g$ indicates gas-phase properties, $c v$ is the control volume, $c s$ is the control surface. Equation 4 is a form of Darcy's law.

Equations 1 and 2 are solved implicitly on an arbitrary contracting grid using Landau coordinates with a non-linear Newton's method. Equation 3 is solved analytically, and Equation 4 is solved explicitly for $v_{g}$ and directly integrated in the gas-phase continuity equation. MOPAR has been validated using both experimental data and code-to-code comparisons, and additional details can be found in Refs. 4-6.

\section{Coupling Aspects}

The interaction between the hypersonic gas flow and the surface of a planetary entry vehicle can be simulated in LeMANS by solving the species mass balance,

$$
-\rho_{w} D_{k, w} \nabla Y_{k, w}+\rho_{w} v_{w} Y_{k, w}=M_{k} \dot{w}_{k}
$$


momentum balance,

$$
p_{n}+\rho_{n} v_{n}^{2}=p_{w}+\rho_{w} v_{w}^{2}
$$

and energy balance,

$$
q_{c o n v}+\dot{m}_{c} h_{c, w}+\dot{m}_{g} h_{g, w}=\left(1-\alpha_{w}\right) q_{r a d}+\sigma \epsilon\left(T_{w}^{4}-T_{\infty}^{4}\right)+\left(\dot{m}_{c}+\dot{m}_{g}\right) h_{w}+q_{c o n d}
$$

equations to obtain the species mass fractions $\left(Y_{k, w}\right)$, gas density $\left(\rho_{w}\right)$, and gas normal velocity $\left(v_{w}\right)$. These equations require additional unknowns which can be calculated using the FRSC Module and MOPAR. The radiative heat flux $q_{r a d}$ in the energy balance equation is neglected in the current study.

The species production rates at the surface are given by,

$$
\dot{w}_{k}=\dot{w}_{k, c}+\frac{Y_{k, g}}{M_{k}} \dot{m}_{g}
$$

The first term in Equation 8 accounts for the contribution from the surface reactions and is calculated by the FRSC Module based on the pressure, temperature, and species concentrations at the wall. The second term accounts for the production of gaseous species from pyrolysis gases and is calculated by MOPAR. The mass fractions, $Y_{k, g}$, appearing in the second term of Equation 8 are for the pyrolysis gas mixture and not the gaseous mass fractions at the surface.

The mass blowing rate at the surface includes both the contribution of the removal of bulk species by surface reactions calculated by the FRSC Module, and the rate of pyrolysis gases passing through the surface obtained from MOPAR. This blowing rate is given by,

$$
\dot{m}=\rho_{w} v_{w}=\dot{m}_{c}+\dot{m}_{g}=-\sum_{j=1}^{N B} M_{j} \dot{w}_{j}+\dot{m}_{g}
$$

The recession rate is calculated in LeMANS using the mass blowing rate due to surface reactions (char) as,

$$
\dot{s}=\frac{\dot{m}_{c}}{\rho_{b}}
$$

where $\rho_{b}$ is the mass density of the bulk environment (char).

The coupling between LeMANS and MOPAR is presented schematically in Figure 1. The coupled simulations begin with the steady-state cold wall non-ablating solution obtained using LeMANS from an initial trajectory point. MOPAR is first called from LeMANS to calculate the surface temperature, pyrolysis gas mass blowing rate, and pyrolysis mixture composition based on the conduction heat flux (equal to the convective heat flux for this initial point), recession rate (equal to zero for this initial point), and pressure along the surface. MOPAR then passes these values to LeMANS, where the flow field mesh is first moved based on the surface recession rate, and then the fluid governing equations are solved iteratively for a user-defined number of iterations. The species mass and momentum balance equations are solved iteratively using Newton's method during each global iteration, while the energy balance equation is solved for the conduction heat flux $q_{\text {cond }}$. Then, MOPAR is called again, and the updated as well as the initial values of conduction heat flux, recession rate, and surface pressure are used as boundary conditions to solve the time-accurate governing equations. This process is repeated until a converged steady-state solution is reached at the current trajectory point.

MOPAR has been recently modified to simulate nonequilibrium chemical processes for the pyrolysis gas. However, it was recently shown that the lack of appropriate chemistry models for the pyrolysis gas prevents this feature from providing reasonable results. ${ }^{19}$ Therefore, two different pyrolysis gas assumptions are considered in this study. The first assumes that the pyrolysis gas is in chemical equilibrium. The second assumes that the pyrolysis gas is chemically "frozen" and does not react with any gas-phase species. In the first assumption, the properties of the pyrolysis gas are determined as functions of temperature using look-up tables generated by assuming chemical equilibrium. The atomistic mass fractions of the gas are determined by the resin decomposition since the decomposing solid is assumed to be transformed to the pyrolysis gas. In the second approach, the properties of the pyrolysis gas are calculated using curve fit relations similar to the ones employed in LeMANS (i.e. viscosity curve fits and Eucken's relation). These two assumptions provide an opportunity to investigate the effects of the pyrolysis gas composition on the flow field predictions for a heat-shield with a charring ablator. 


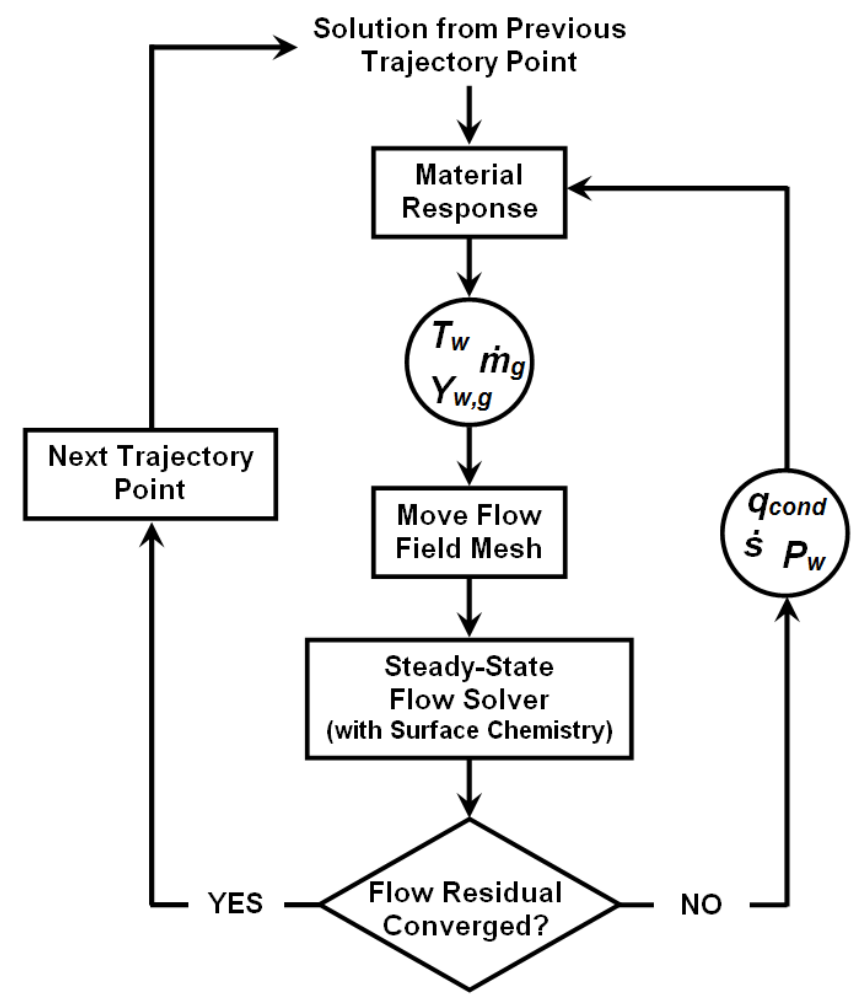

Figure 1. Coupling scheme for between LeMANS and MOPAR.

\section{Effects of Pyrolysis Gas Chemistry}

\section{A. Numerical Setup}

In order to demonstrate the coupling between LeMANS and MOPAR, numerical simulations are performed based on the entry conditions of the Stardust sample return capsule (SRC). The Stardust payload was launched in 1999 on a mission to collect samples from interstellar dust and the tail of the Comet Wild-2, and return them to Earth. The SRC, shown schematically in Figure 2(a), landed in the Utah desert in January of 2006. The Earth entry trajectory of the return capsule is presented in Figure 2(b). The Stardust spacecraft then continued its travel through the solar system, on a mission to image Comet Tempel-1. It was decommissioned after completing that final mission in March 2011. The Stardust mission represents the first ever return of a sample from a comet; a significant milestone in the human exploration of space. With an entry velocity of $12.6 \mathrm{~km} / \mathrm{s}$, the capsule was also the fastest man-made object ever to enter the Earth's atmosphere, providing a unique test case to evaluate numerical simulations. In order to protect the vehicle from the extreme entry conditions, the thermal protection system for the Stardust capsule used the phenolicimpregnated carbon ablator (PICA), ${ }^{20}$ which is a lightweight material with a density of approximately 240 $\mathrm{kg} / \mathrm{m}^{3}$.

The freestream conditions simulated in this study are listed in Table $1 .{ }^{21}$ The $81 \mathrm{~km}$ trajectory point is used to initialize the coupled simulations. The gas environment chemistry model used in this study was originally developed by Martin and Boyd, ${ }^{22}$ and consists of the following 38 species:

$$
\begin{gathered}
\mathrm{N}_{2}, \mathrm{O}_{2}, \mathrm{NO}, \mathrm{N}, \mathrm{O}, \mathrm{N}_{2}^{+}, \mathrm{O}_{2}^{+}, \mathrm{NO}^{+}, \mathrm{N}^{+}, \mathrm{O}^{+}, \mathrm{e} \\
\mathrm{C}, \mathrm{C}_{2}, \mathrm{C}_{3}, \mathrm{CO}, \mathrm{CO}_{2}, \mathrm{CN}, \mathrm{NCO}, \mathrm{CO}^{+}, \mathrm{CN}^{+}, \mathrm{C}^{+}
\end{gathered}
$$

$$
\mathrm{H}, \mathrm{H}_{2}, \mathrm{OH}, \mathrm{H}_{2} \mathrm{O}, \mathrm{HO}_{2}, \mathrm{H}_{2} \mathrm{O}_{2}, \mathrm{NH}, \mathrm{HNO}, \mathrm{CH}, \mathrm{CH}_{2}, \mathrm{CH}_{3}, \mathrm{CH}_{4}, \mathrm{C}_{2} \mathrm{H}, \mathrm{C}_{2} \mathrm{H}_{2}, \mathrm{HCN}, \mathrm{HCO}, \mathrm{H}^{+}
$$

The material of the thermal protection system used in the current study is TACOT (Theoretical Ablative Composite for Open Testing). ${ }^{23}$ TACOT is a fictitious material with properties similar to carbon-phenolic materials such as PICA, and has been used for ablation code benchmarks. Two different assumptions are used 


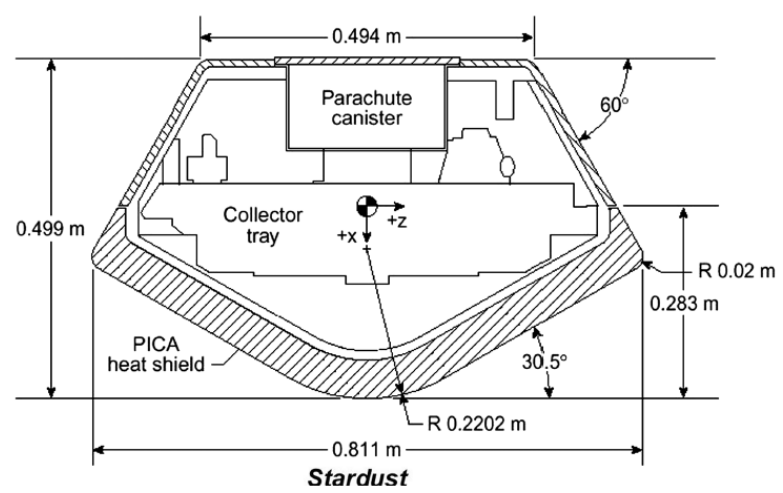

(a) Geometry

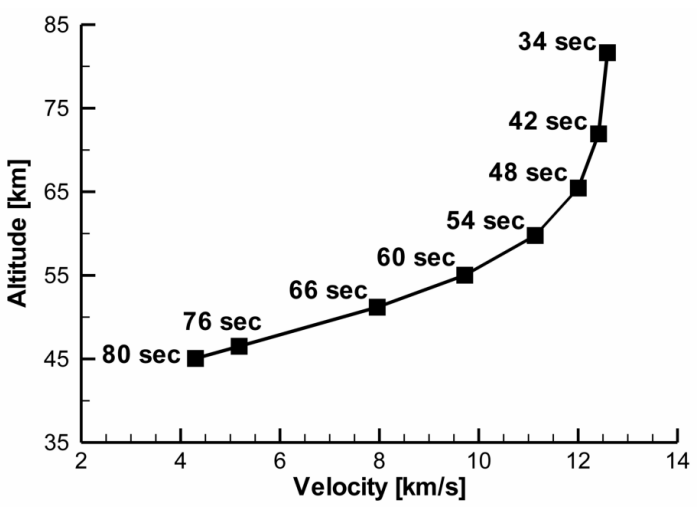

(b) Trajectory

Figure 2. Stardust sample return capsule geometry and entry trajectory.

Table 1. Freestream conditions. ${ }^{21}$

\begin{tabular}{ccccc}
\hline $\begin{array}{c}\text { Time from } \\
\text { entry, sec }\end{array}$ & $\begin{array}{c}\text { Altitude, } \\
\mathrm{km}\end{array}$ & $\begin{array}{c}\text { Velocity, } \\
\mathrm{m} / \mathrm{sec}\end{array}$ & $\begin{array}{c}\text { Density, } \\
\mathrm{kg} / \mathrm{m}^{3}\end{array}$ & $\begin{array}{c}\text { Temperature, } \\
\mathrm{K}\end{array}$ \\
\hline 34 & 81.0 & 12,385 & $1.3 \times 10^{-5}$ & 217.6 \\
36 & 78.5 & 12,337 & $1.9 \times 10^{-5}$ & 218.1 \\
38 & 76.0 & 12,269 & $2.7 \times 10^{-5}$ & 218.7 \\
40 & 73.5 & 12,181 & $3.9 \times 10^{-5}$ & 220.0 \\
\hline
\end{tabular}

for the pyrolysis gas composition. The first approach assumes that the pyrolysis gas is in chemical equilibrium. The second approach assumes that the pyrolysis gas is composed entirely of chemically "frozen" phenol gas $\left(\mathrm{C}_{6} \mathrm{H}_{5} \mathrm{OH}\right)$. The chemical mechanisms that are assumed to occur at the surface of the entry capsule are presented in Table 2. These mechanisms were developed by Driver et al. by comparing CFD predictions to heat transfer and recession rate measurements obtained at an arc-jet facility for PICA. ${ }^{24,25}$ The mechanisms found by Driver et al. include the oxidation of bulk carbon by atomic oxygen with a constant reaction efficiency of $\gamma=0.90$, and the oxidation of bulk carbon by molecular oxygen with a constant efficiency of $\gamma=0.01$. These mechanisms also include the recombination of atomic nitrogen at the surface with a constant recombination efficiency of $\gamma=0.05$. These reactions are exothermic and the heat released by each reaction are also presented in Table 2 .

Table 2. Surface chemical reactions. ${ }^{24,25}$

\begin{tabular}{ccc}
\hline$\#$ & Reaction & Efficiency \\
\hline 1 & $\mathrm{O}+\mathrm{C}(\mathrm{b}) \rightarrow \mathrm{CO}, \Delta H=360 \mathrm{~kJ} / \mathrm{mol}$ & 0.90 \\
2 & $\mathrm{O}_{2}+2 \mathrm{C}(\mathrm{b}) \rightarrow 2 \mathrm{CO}, \Delta H=217 \mathrm{~kJ} / \mathrm{mol}$ & 0.01 \\
3 & $\mathrm{~N}+\mathrm{N} \rightarrow \mathrm{N}_{2}, \Delta H=950 \mathrm{~kJ} / \mathrm{mol}$ & 0.05 \\
\hline
\end{tabular}

Figure 3 presents the computational grid used in the flow field simulations for this study. The grid is axisymmetric and is composed of approximately 30,000 quadrilateral cells, with approximately 200 cells in the axial direction and 150 cells in the radial direction along the Stardust forebody. Clustering is used near the capsule surface and the size of the first cell away from the wall is $10^{-6} \mathrm{~m}$. Note that the computational domain considered in this study does not include the sharp shoulder of the capsule since MOPAR is a one-dimensional code. 


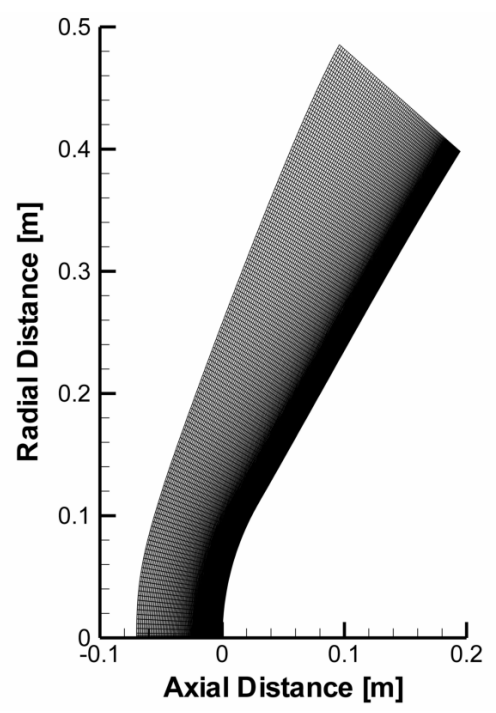

(a) Overview

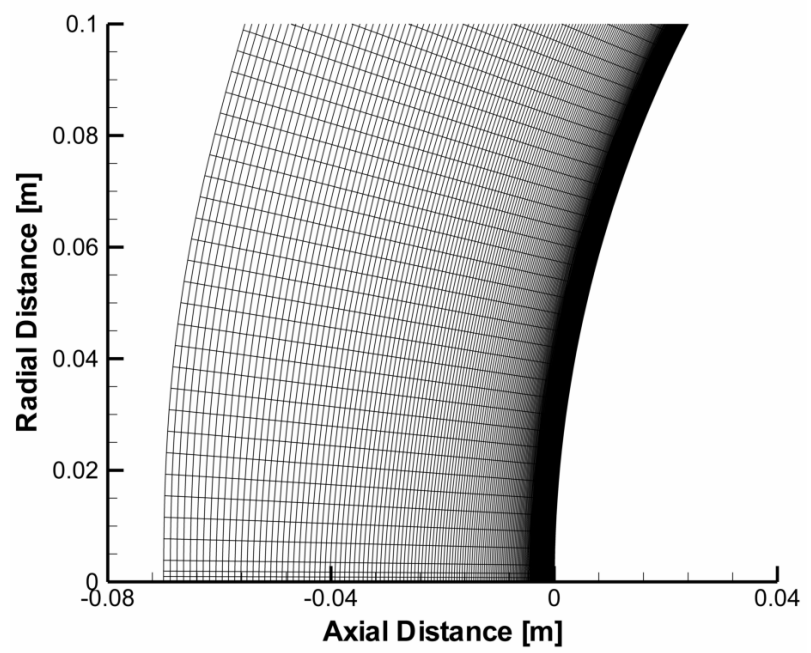

(b) Stagnation Region

Figure 3. Computational grid used in the flow field.

\section{B. Numerical Results}

The main goal of this study is to couple a viscous Navier-Stokes solver to a one-dimensional material response code. This coupling can account for nonequilibrium chemical processes that may occur at the surface of an atmospheric entry vehicle. This section presents a demonstration of this coupling by considering the re-entry conditions of the Stardust sample return capsule. Two sets of simulations are performed at three different trajectory points. The first set assumes that the pyrolysis gas is in chemical equilibrium, while the second set assumes that the pyrolysis gas is non-reacting phenol.

Figure 4 presents the temperature in the flow field predicted by LeMANS and within the solid predicted by MOPAR. The results obtained by assuming that the pyrolysis gas is in chemical equilibrium are on top, and the results predicted using the "frozen" phenol gas are on the bottom. As the altitude decreases, the peak temperature in the flow field decreases, while the temperature within the solid increases as heat propagates inside the TPS material. The figures also show that heat propagates farther inside the solid for the chemically frozen phenol compared to the equilibrium gas, and as a result, the temperature within the solid is larger.

Temperature profiles along the surface of the Stardust forebody are presented in Figure 5 for the three trajectory points considered in this study. The solid lines represent the solutions obtained by assuming that the pyrolysis gas is in chemical equilibrium, while the dashed lines are the solutions obtained by assuming that the pyrolysis gas is non-reacting phenol $\left(\mathrm{C}_{6} \mathrm{H}_{5} \mathrm{OH}\right)$. As the altitude decreases, the surface temperature increases for both assumptions. The temperature at the stagnation point increases from about $1600 \mathrm{~K}$ to $2100 \mathrm{~K}$ for the equilibrium pyrolysis gas, and from about $1850 \mathrm{~K}$ to $2350 \mathrm{~K}$ for the non-reacting pyrolysis gas. This shows that the non-reacting pyrolysis gas assumption produces an overall larger surface temperature compared to the equilibrium gas assumption for the trajectory conditions considered in this study. Specifically, the difference in the temperature at the stagnation point predicted using the two assumptions is approximately $15 \%$.

The temperatures predicted using both pyrolysis gas assumptions are smaller than some of the values published in other studies. For instance, a study by Winter and Trumble ${ }^{26}$ deduced from radiation measurements obtained during the Stardust re-entry average temperatures from about $2100 \mathrm{~K}$ to $2600 \mathrm{~K}$ and peak temperatures from about $2300 \mathrm{~K}$ to $2900 \mathrm{~K}$ for the trajectory altitudes considered in the current study. One possible cause of this difference is the fact that the current study assumes that the TPS material is TACOT, instead of PICA. Another possible cause can be attributed to the trajectory simulation approach undertaken in this study. By using the $81.0 \mathrm{~km}$ altitude conditions as the initial solution for the coupled simulations, it is implicitly assumed that significant heat transfer to the surface does not start until this point. However, a study by Boyd et al. showed that the aerodynamic heating is significant at the $81.0 \mathrm{~km}$ trajectory point. ${ }^{27}$ 


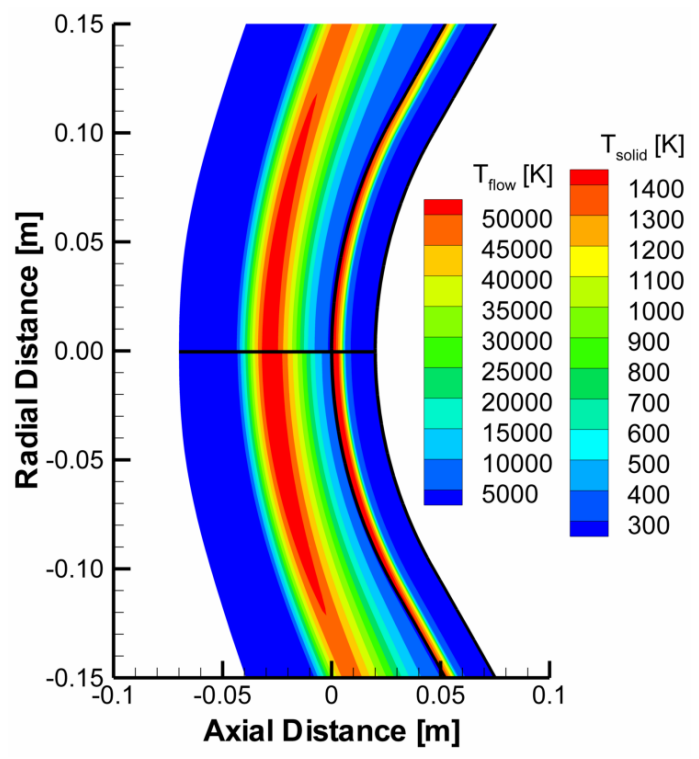

(a) $\mathrm{t}=36 \mathrm{sec}(78.5 \mathrm{~km})$

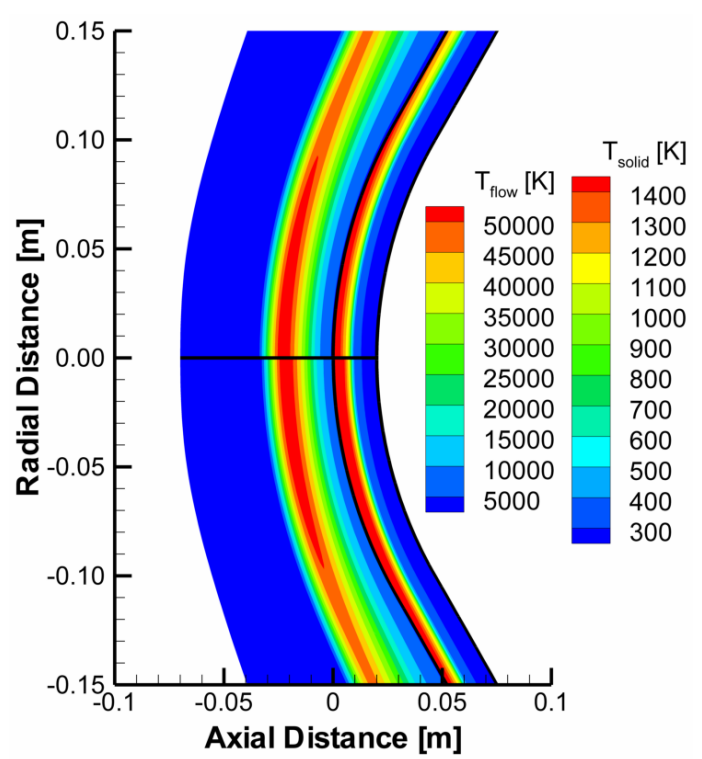

(b) $\mathrm{t}=38 \mathrm{sec}(76.0 \mathrm{~km})$

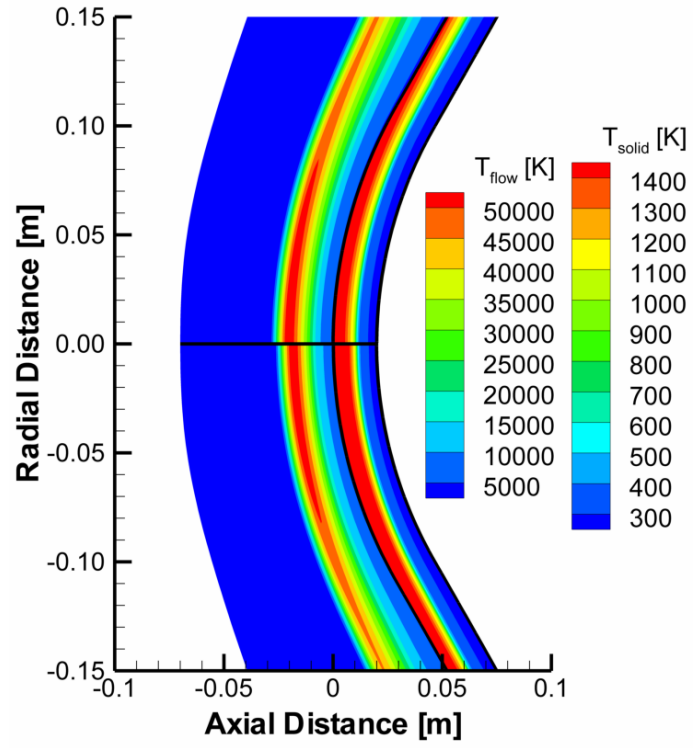

(c) $\mathrm{t}=40 \mathrm{sec}(73.5 \mathrm{~km})$

Figure 4. Flow field and solid temperature contours for equilibrium pyrolysis gas (top) and non-reacting phenol (bottom). 


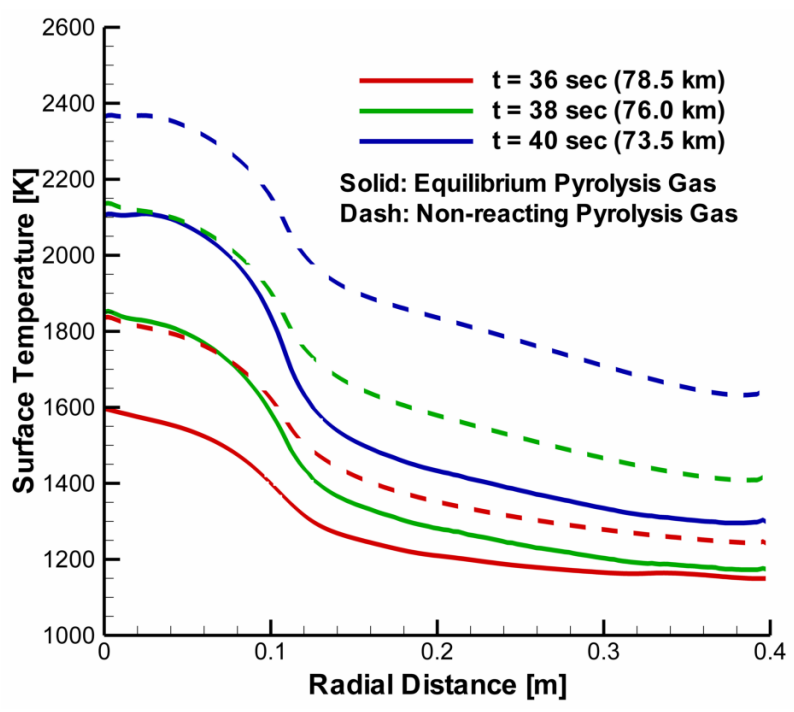

Figure 5. Temperature distribution along the Stardust forebody.

As a result, this assumption can affect the predicted surface temperatures along the subsequent trajectory points. One potential remedy for this drawback is to simply start the coupled simulations at an earlier trajectory point. However, this may be difficult to achieve because the continuum assumption may not be valid and the Navier-Stokes equations may not be physically accurate at these higher altitudes.

The convective heat transfer along the Stardust forebody is shown in Figure 6. This heat flux is made up of contributions from the translational/rotational energy mode, the vibrational/electronic/electron energy mode, and the mass diffusion of enthalpy to and from the surface. These components are also presented in Figure 6. Overall, the convective heat fluxes follow similar trends to those observed in the surface temperature. Namely, as the altitude of the trajectory points considered in this study decreases, the convective heat transfer to the vehicle increases for both the equilibrium and non-reacting pyrolysis gas assumptions. Figure 6 (a) shows that the non-reacting pyrolysis gas assumption increases the convective heat transfer relative to the equilibrium gas assumption by about $50 \%$ for the $78.5 \mathrm{~km}$ point and about $30 \%$ for the $73.5 \mathrm{~km}$ point. This increase is due to the diffusive component, which accounts for the transport of enthalpy across the boundary layer and is affected by chemical reactions that occur at the surface. The diffusive heat flux calculated using the non-reacting phenol gas assumption is overall larger than the values obtained with the equilibrium assumption, which indicates that the heat released by the surface reactions (i.e. carbon oxidation and nitrogen recombination) is larger for the non-reacting phenol gas assumption. The convective heat flux component due to the translational/rotational mode is larger for the equilibrium pyrolysis gas assumption compared to the non-reacting gas assumption for the $76.0 \mathrm{~km}$ and $73.5 \mathrm{~km}$ altitude conditions. The vibrational/electronic/electron component is relatively small for both assumptions at the conditions considered in this study.

The removal of charred carbon due to the oxidation reactions and the flow of pyrolysis gas across the surface produce a mass blowing along the Stardust forebody. Figure 7 presents the total mass blowing along the forebody surface. The figure also presents the components due to the flow of pyrolysis gas and the consumption of charred carbon by the oxidation reactions. Figure 7(a) shows that the total mass blowing rate calculated using the non-reacting pyrolysis gas assumption is overall larger than the rate obtained with the equilibrium pyrolysis gas assumption. One interesting result is that the total blowing rate actually decreases when the altitude decreases from $78.5 \mathrm{~km}$ to $76.0 \mathrm{~km}$ for both pyrolysis gas assumptions. This result is counter-intuitive since the blowing rate is expected to actually increase because the heat flux and surface temperature increase. However, as previously mentioned, since the solution for the $81.0 \mathrm{~km}$ is used as the starting point for this trajectory analysis, it is implicitly assumed that the ablation process does not begin until some point right after the $81.0 \mathrm{~km}$ point. As a result, the blowing rate reaches a peak at a time between the $81.0 \mathrm{~km}$ and $78.5 \mathrm{~km}$ points, decreases once the ablation process begins, and then increases due to the increase in heat flux and temperature. This is physically unrealistic and similar to the overall 


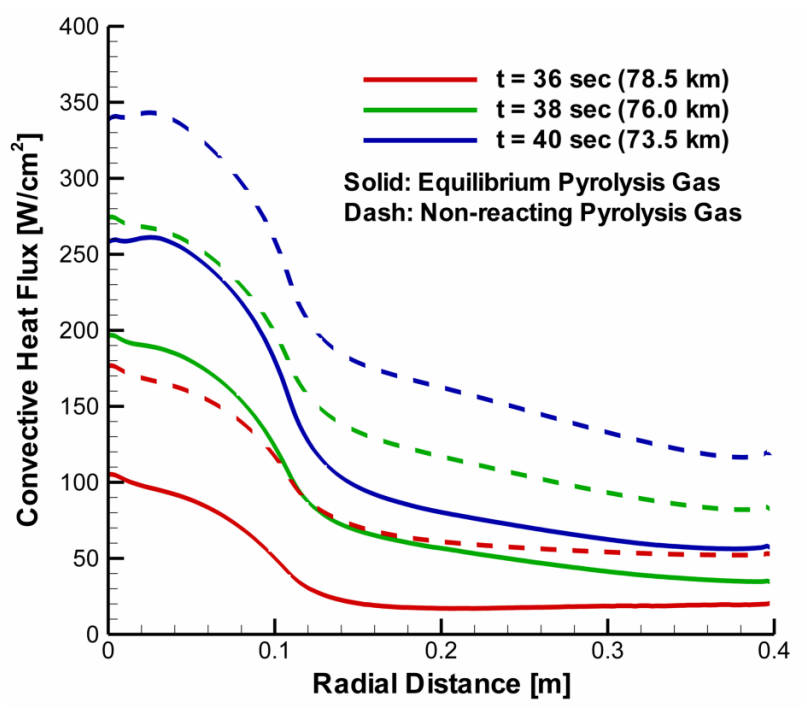

(a) Total

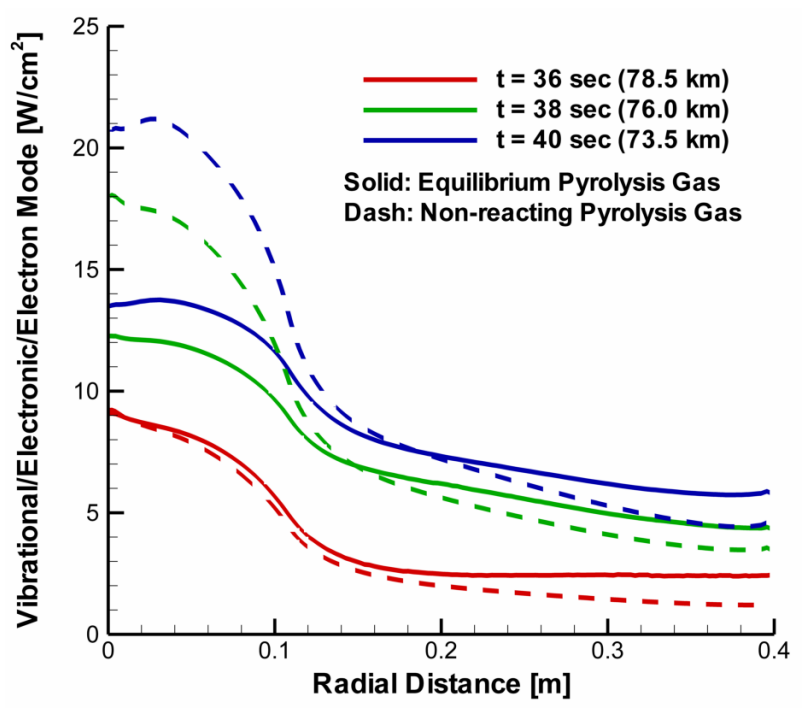

(c) Vibrational/electronic/electron mode

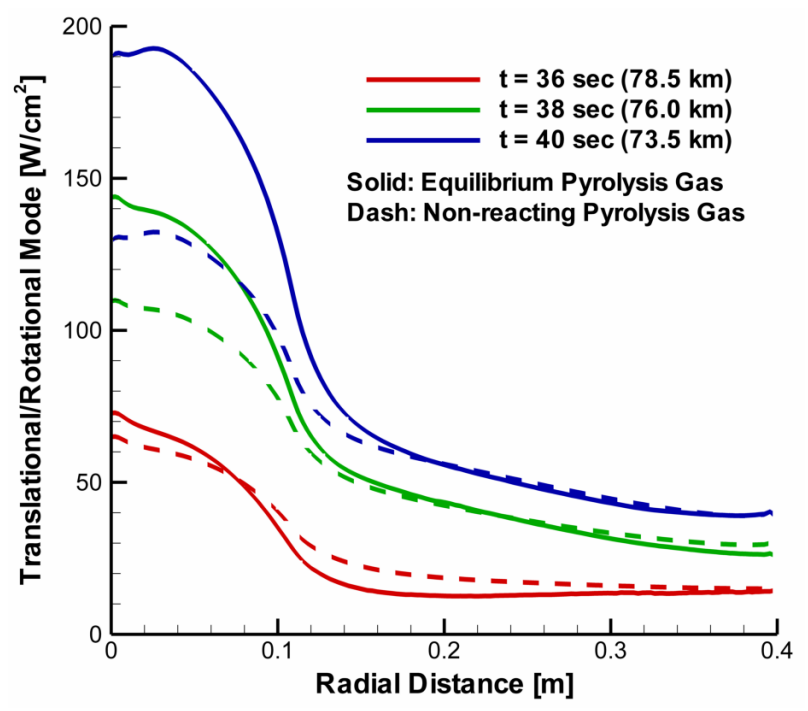

(b) Translational/rotational mode

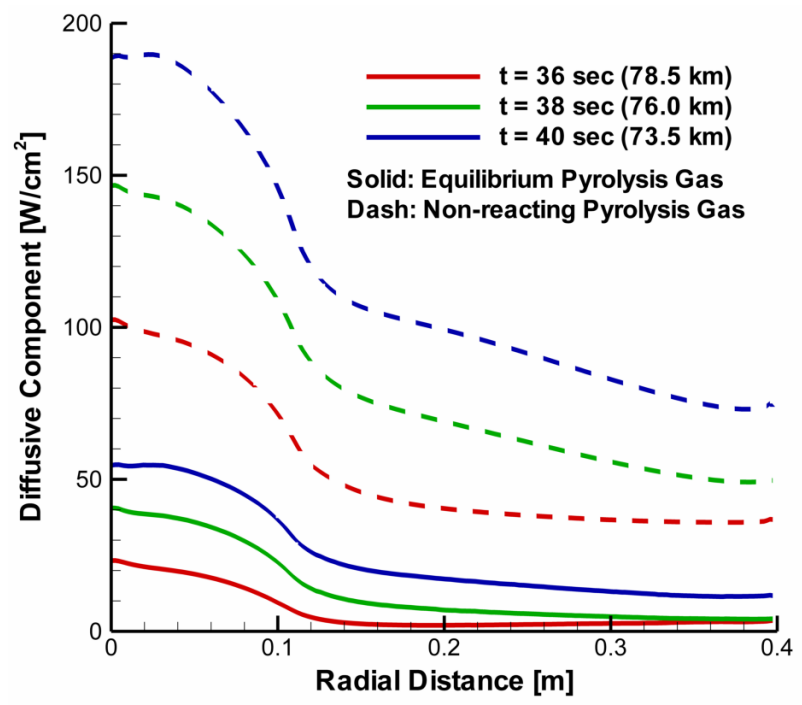

(d) Diffusive

Figure 6. Convective heat transfer components along the Stardust forebody. 
low surface temperatures, may be a consequence of how the coupled simulations were performed. A more accurate approach would require the coupled simulations to start at a higher trajectory altitude. However, this may be difficult because the continuum assumption may not be valid at these higher points.

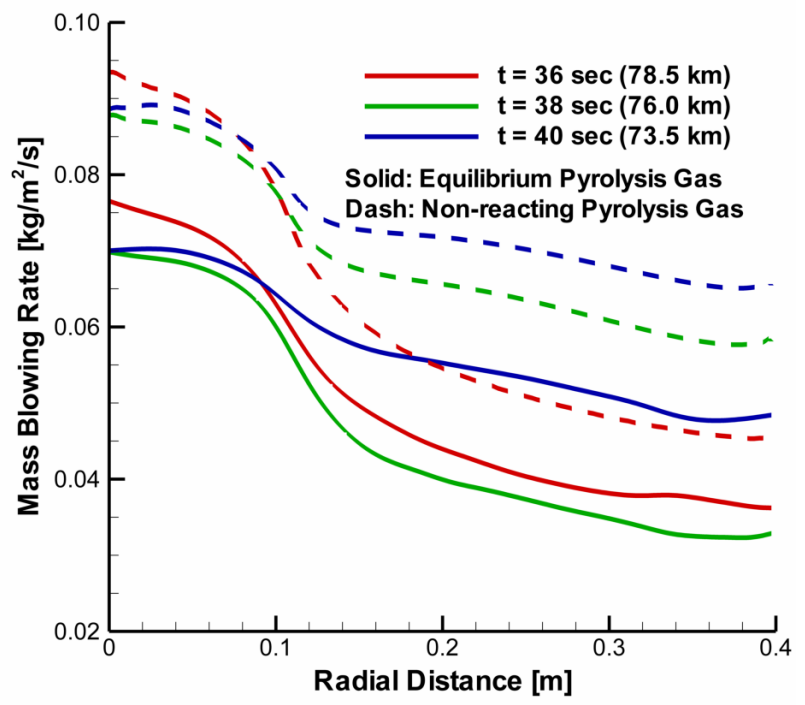

(a) Total

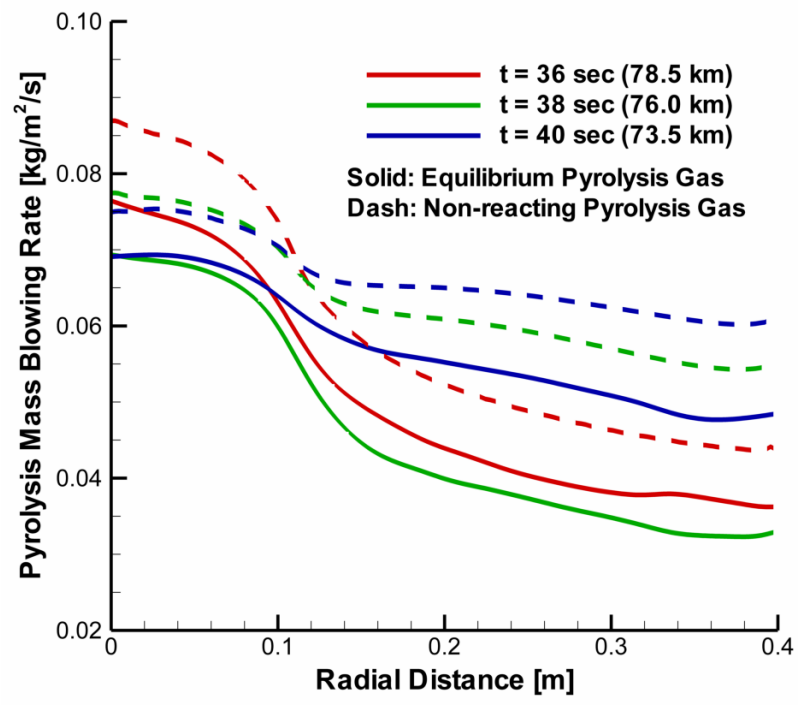

(b) Pyrolysis

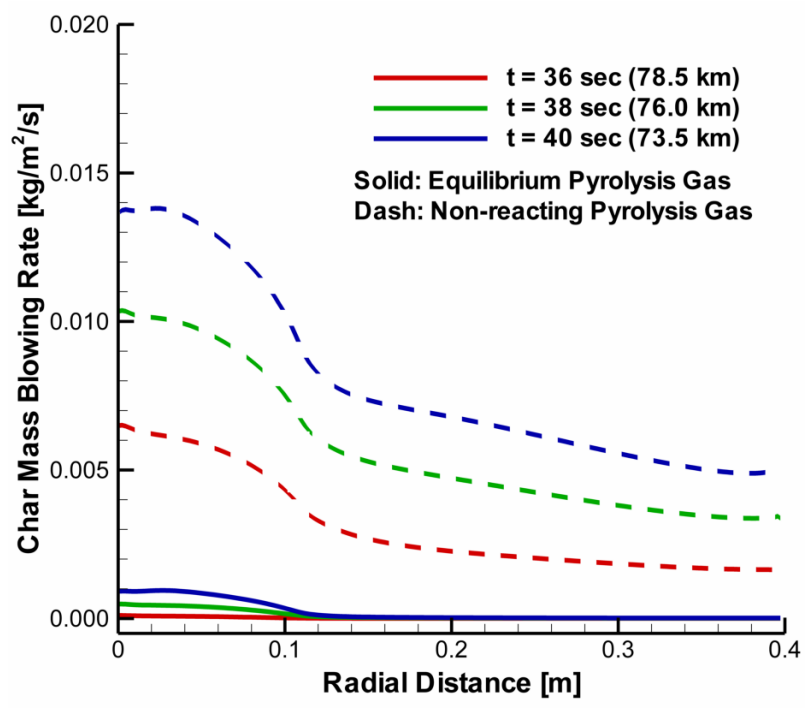

(c) Char

Figure 7. Mass blowing rate components along the Stardust forebody.

Figure 7 also shows that the mass blowing rate due to the removal of bulk carbon (char) calculated using the non-reacting pyrolysis gas assumption is well over an order of magnitude larger than the rate predicted using the equilibrium gas assumption. This indicates that the rates of the carbon oxidation reactions at the surface for the non-reacting pyrolysis gas cases are larger than the rates for the equilibrium gas cases. The reason for this is that the added species produced by the pyrolysis gas react with the nitrogen and oxygen atoms in the gas-phase, which can either decrease (in the case of atom consumption) or increase (in the case of atom production) the concentration of these atoms in the boundary layer. Figure 8 presents the species number densities along the stagnation line for the $78.5 \mathrm{~km}$ and $73.5 \mathrm{~km}$ conditions. The densities of both atomic oxygen and atomic nitrogen are much smaller for the equilibrium case than for the non-reacting phenol case. This is due to the fact that both atomic oxygen and atomic nitrogen interact with the pyrolysis gas, which is composed mainly of $\mathrm{C}_{2} \mathrm{H}_{2}, \mathrm{CO}$, and $\mathrm{H}_{2}$ as can be seen in Figure 9. These chemical interactions form new species, such as $\mathrm{OH}$ and $\mathrm{NH}$. As a result, fewer oxygen and nitrogen atoms participate in surface 
reactions, which changes the gas-phase composition in the boundary layer, and decreases both the mass blowing rate due to char removal, as well as the diffusive and convective heat transfer to the vehicle surface. Although not shown, the results for the $76.0 \mathrm{~km}$ point also follow similar trends.
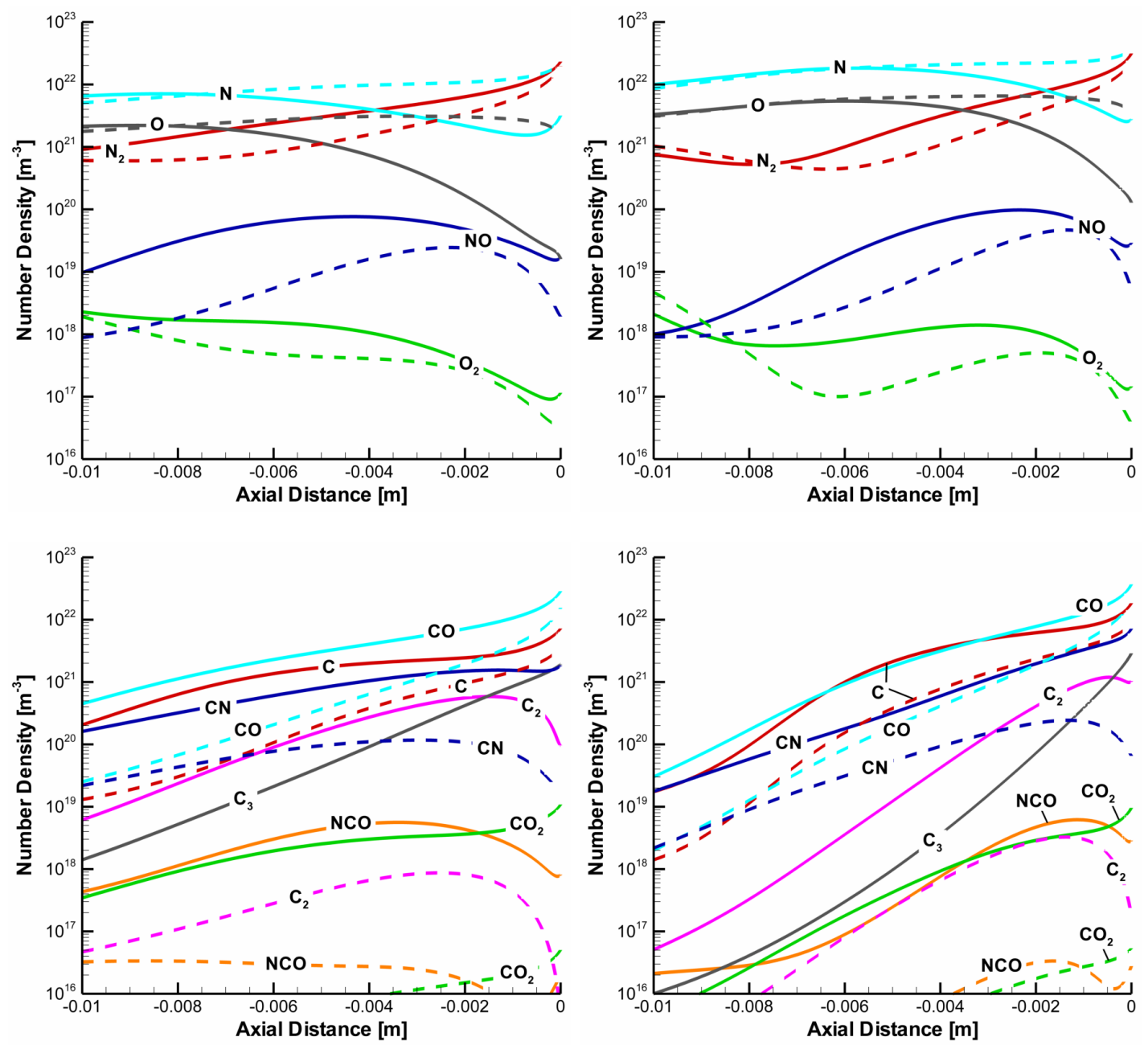

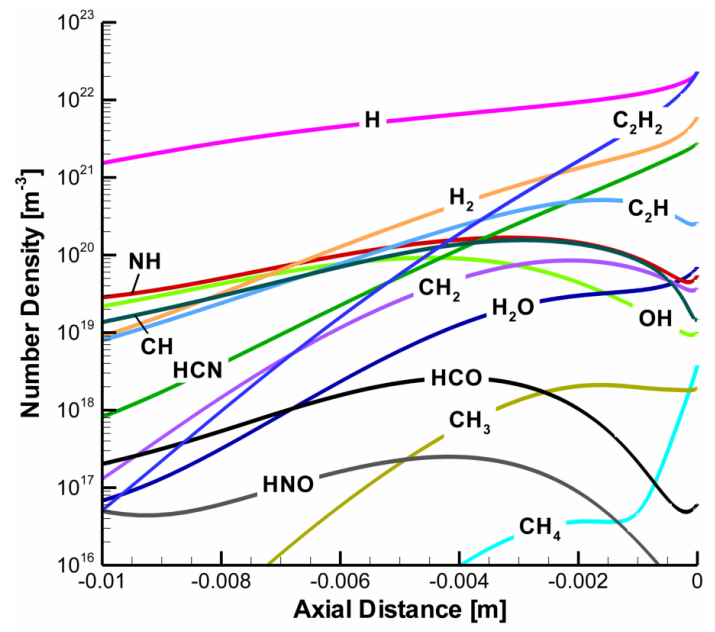

(a) $\mathrm{t}=36 \sec (78.5 \mathrm{~km})$

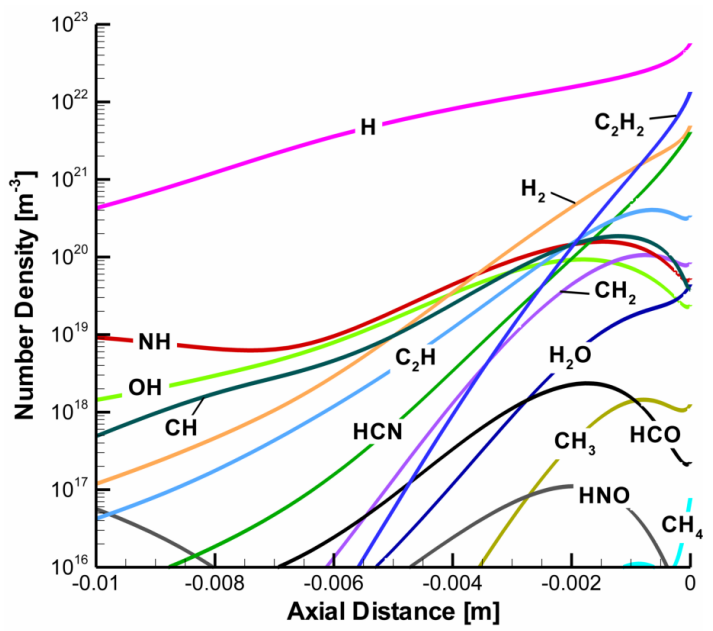

(b) $\mathrm{t}=40 \sec (73.5 \mathrm{~km})$

Figure 8. Species number densities along the stagnation line for the equilibrium pyrolysis gas assumption (solid) and non-reacting pyrolysis gas (dash). 


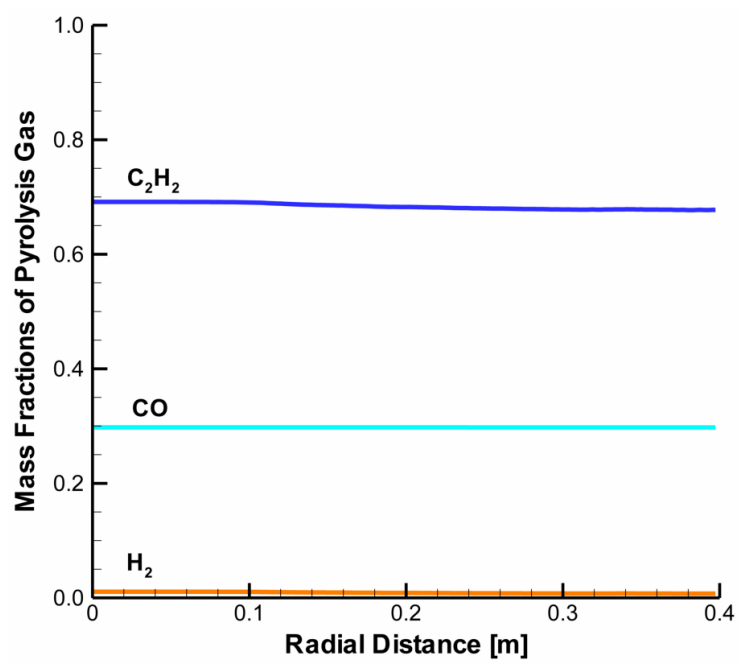

(a) $\mathrm{t}=36 \mathrm{sec}(78.5 \mathrm{~km})$

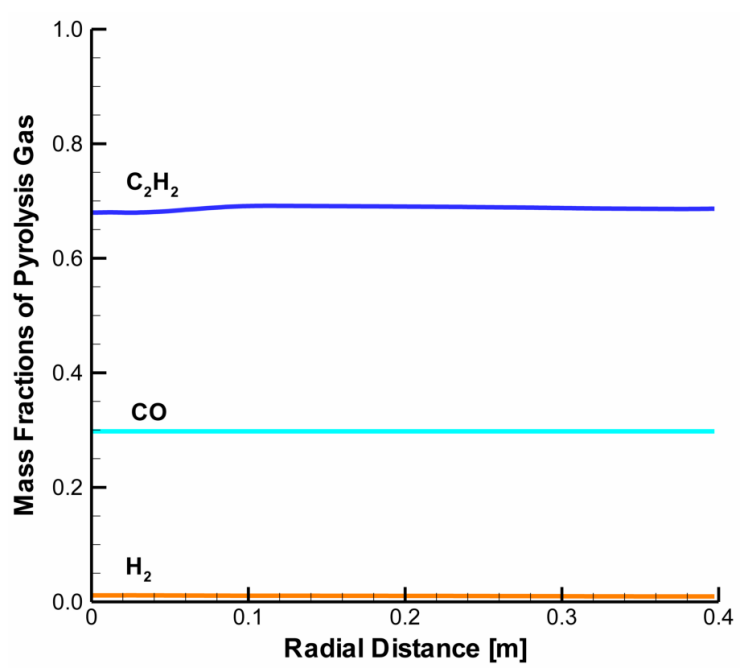

(b) $\mathrm{t}=40 \mathrm{sec}(73.5 \mathrm{~km})$

Figure 9. Mass fractions of the pyrolysis gas along the Stardust forebody for the chemical equilibrium approach.

\section{Conclusion}

This paper described a coupling scheme between a Navier-Stokes solver and a material response code. The coupling accounts for nonequilibrium gas-surface reactions. The Navier-Stokes solver used in this study is LeMANS, which is a three-dimensional CFD code that simulates hypersonic reacting flows with thermochemical nonequilibrium effects. The material response code is called MOPAR, which is a one-dimensional control volume finite-element code that models heat conduction and pyrolysis gas behavior with inner decomposition. In the coupling scheme used in this study, the conductive heat flux, surface pressure, and recession rate are calculated in LeMANS and used in MOPAR to obtain the surface temperature, mass blowing rate due to pyrolysis gas, and pyrolysis gas mixture composition. This exchange of information occurs at user-defined number of iterations, and the process continues until the flow field solution reaches a steady-state. The capabilities of the newly developed coupled tool were demonstrated using a test case based on the re-entry conditions of the Stardust sample return capsule. Coupled simulations were performed at three different trajectory altitudes between $78.5 \mathrm{~km}$ and $73.5 \mathrm{~km}$. The effects of the pyrolysis gas chemistry were evaluated by using two different assumptions. The pyrolysis gas is assumed to be in chemical equilibrium for the first approach and non-reacting chemically "frozen" phenol for the second approach.

The equilibrium pyrolysis gas assumption produced overall lower surface temperatures, convective heat fluxes, and mass blowing rates compared to the non-reacting pyrolysis gas approach for the trajectory conditions considered in this study. The reason for this is that the additional species produced by the pyrolysis gas in chemical equilibrium react with oxygen and nitrogen atoms in the gas-phase to form new species, such as $\mathrm{OH}$ and $\mathrm{NH}$. As a result, fewer oxygen and nitrogen atoms participate in surface reactions, which decreases the convective heat flux. This decrease in the convective heat flux causes a decrease in both the conductive heat flux and surface temperature relative to the non-reacting pyrolysis gas approach.

This study demonstrated the ability to couple a Navier-Stokes solver and a material response code to account for nonequilibrium gas-surface interactions. In future work, this approach must be validated by comparing the numerical results with experimental data, such as thermocouple temperature measurements obtained in arc-jet facilities. Appropriate chemical models are also needed for the pyrolysis gas in order to truly simulate nonequilibrium processes. Finally, a complete trajectory analysis will be performed to evaluate the effects of nonequilibrium gas-surface interactions for various planetary entry conditions.

\section{Acknowledgments}

The authors thank Dr. Matthew MacLean from CUBRC Inc. for sharing the finite-rate surface chemistry module used in this work. The authors gratefully acknowledge funding for this work through NASA SBIR 
Phase II Contract NNX11CA27C. The use of supercomputers through the NASA Advanced Supercomputing Division was essential to this work and is also greatly appreciated.

\section{References}

\footnotetext{
${ }^{1}$ Scalabrin, L. C., Numerical Simulation of Weakly Ionized Hypersonic Flow over Reentry Capsules, Ph.D. thesis, University of Michigan, 2007.

${ }^{2}$ Martin, A., Scalabrin, L. C., and Boyd, I. D., "High Performance Modeling of Atmospheric Re-entry Vehicles," Journal of Physics: Conference Series, Vol. 341, No. 1, 2012, Article 012002.

${ }^{3}$ Alkandry, H., Farbar, E. D., and Boyd, I. D., "Evaluation of Finite-Rate Surface Chemistry Models for Simulation of the Stardust Reentry Capsule," AIAA Paper 2012-2874, June 2012.

${ }^{4}$ Martin, A. and Boyd, I. D., "Non-Darcian Behavior of Pyrolysis Gas in a Thermal Protection System," Journal of Thermophysics and Heat Transfer, Vol. 24, No. 1, January-March 2010, pp. 60-68.

${ }^{5}$ Martin, A. and Boyd, I. D., "Implicit Implementation of Material Response and Moving Meshes for Hypersonic Re-Entry Ablation," AIAA Paper 2009-670, January 2009.

${ }^{6}$ Martin, A. and Boyd, I. D., "Strongly Coupled Computation of Material Response and Nonequilibrium Flow for Hypersonic Ablation," AIAA Paper 2009-3597, June 2009.

${ }^{7}$ Wilke, C. R., "A Viscosity Equation for Gas Mixtures," Journal of Chemical Physics, Vol. 18 No. 4, 1950, pp. 517-519.

${ }^{8}$ Blottner, F. G., Johnson, M., and Ellis, M., "Chemically Reacting Viscous Flow Program for Multi-Component Gas Mixtures," Tech. rep., SC-RR-70-754, Sandia Laboratories, Albuquerque, New Mexico, 1971.

${ }^{9}$ Vincenti, W. G. and Kruger, C. H., Introduction to Physical Gas Dynamics, Krieger Publishing Company, 2002.

${ }^{10}$ Sutton, K. and Gnoffo, P. A., "Multi-Component Diffusion with Application to Computational Aerothermodynamics," AIAA Paper 1998-2575, June 1998.

${ }^{11}$ MacCormack, R. W. and Candler, G. V., "The Solution of the Navier-Stokes Equations using Gauss-Seidel Line Relaxation," Computers and Fluids, Vol. 17, 1989, pp. 135-150.

${ }^{12}$ Martin, A. and Boyd, I. D., "Mesh Tailoring for Strongly Coupled Computation of Ablative Material in Nonequilibrium Hypersonic Flow," AIAA Paper 2010-5062, June 2010.

${ }^{13}$ Zhang, H., Reggio, M., Trepanier, J. Y., and Camarero, R., "Discrete Form of the GCL for Moving Meshes and its Implementation in CFD Schemes," Computers and Fluids, Vol. 22, No. 1, January 1993, pp. 9-23.

${ }^{14}$ Karypis, G. and Kumar, V., "METIS: A Software Package for Partitioning Unstructured Graphs, Partitioning Meshes, and Computing Fill-Reducing Orderings of Sparse Matrices," University of Minnesota, 1998.

${ }^{15}$ Marschall, J. and MacLean, M., "Finite-Rate Surface Chemistry Model, I: Formulation and Reaction System Examples," AIAA Paper 2011-3783, June 2011.

${ }^{16}$ MacLean, M., Marschall, J., and Driver, D. M., "Finite-Rate Surface Chemistry Model, II: Coupling to Viscous NavierStokes Code," AIAA Paper 2011-3784, June 2011.

${ }^{17}$ Amar, A. J., Modeling of One-Dimensional Ablation with Porous Flow Using Finite Control Volume Procedure, Master's thesis, North Carolina State University, 2006.

${ }^{18}$ Blackwell, B. F. and Hogan, R. E., "One-Dimensional Ablation Using Landau Transformation and Finite Control Volume Procedure," Journal of Thermophysics and Heat Transfer, Vol. 8, No. 2, April-June 1994, pp. 282-287.

${ }^{19}$ Martin, A., "Modeling of Chemical Nonequilibrium Effects in a Charring Ablator," AIAA Paper 2013-0301, January 2013.

${ }^{20}$ Tran, H. K., Johnson, C. E., Rasky, D. J., Hui, F. C. L., Hsu, M.-T., and Chen, Y. K., "Phenolic Impregnated Carbon Ablators (PICA) for Discovery class missions," AIAA Paper 1996-1911, June 1996.

${ }^{21}$ Trumble, K. A., Cozmuta, I., Sepka, S., Jenniskens, P., and Winter, M., "Postflight Aerothermal Analysis of Stardust Sample Return Capsule," Journal of Spacecraft and Rockets, Vol. 47, No. 5, September-October 2010, pp. 765-774.

${ }^{22}$ Martin, A. and Boyd, I. D., "CFD Implementation of a Novel Carbon-Phenolic-in-Air Chemistry Model for Atmospheric Re-entry," AIAA Paper 2011-143, January 2011.

${ }^{23}$ Lachaud, J., Martin, A., Cozmuta, I., and Laub, B., "Ablation test-case series 1," 4th AFOSR/SNL/NASA Ablation Workshop, 2010.

${ }^{24}$ Driver, D. M., Olson, M. W., Barnhardt, M. D., and MacLean, M., "Understanding High Recession Rates of Carbon Ablators Seen in Shear Tests in an Arc Jet," AIAA Paper 2010-1177, January 2010.

${ }^{25}$ Driver, D. M. and MacLean, M., "Improved Predictions of PICA Recession in Arc Jet Shear Tests," AIAA Paper 2011-141, January 2011.

${ }^{26}$ Winter, M. W. and Trumble, K. A., "Near-Ultraviolet Emission Spectroscopy During an Airborne Observation of the Stardust Reentry," Journal of Spacecraft and Rockets, Vol. 48, No. 1, January-February 2011, pp. 59-71.

${ }^{27}$ Boyd, I. D., Trumble, K. A., and Wright, M. J., "Modeling of Stardust Entry at High Altitude, Part 1: Flowfield Analysis," Journal of Spacecraft and Rockets, Vol. 47, No. 5, September-October 2010, pp. 708-717.
} 